\title{
Challenges and Successes in Research Work
}

\author{
Elias Westermarck* \\ Department of Clinical Veterinary Sciences, Faculty of Veterinary Medicine, University of Helsinki, Helsinki, Finland
}

${ }^{*}$ Correspondence to: Elias Westermarck, Department of Clinical Veterinary Sciences, Faculty of Veterinary Medicine, University of Helsinki, Helsinki, Finland; E-mail: elias.westermarck@helsinki.fi

Received: April 20, 2018; Accepted: April 29, 2018; Published: May 07, 2018;

\section{Short communication}

After working for ten years as the municipality veterinarian in Ostrobothnia, I took up a job at the College of Veterinary Medicine (Eläinlääketieteellinen korkeakoulu), which is now the Faculty of Veterinary Medicine at the University of Helsinki. Although I was only able to devote a fraction of my working time to science, I recall this time as both interesting and challenging. Most of my university career of 27 years was spent in teaching and administrative work. Over the years, I would find out that carrying out clinical research on small animals is difficult. During my entire career, I only got one period of research leave from my routine work. This was in 1990 when I spent a year in the USA. I could not get a research grant even for my doctoral dissertation. I wrote my synopsis during sick leave after meniscal surgery. The Academy of Finland, Tekes or Sitra all refused to grant funding for research on small animals. In those early days I was somewhat lacking in certain technical skills needed for research work. For example, I did not learn to type before I was 50 years old. Before that, I wrote everything by hand, and my wife typed out the texts. We adopted the same procedure with my doctoral dissertation.

The topics of my research were the consequence of a multitude of coincidences. Much of my research work started without any detailed plan. Often, at the start of the research work I only had a sketchy idea of who might provide funding, who would perform the research work and what the schedule would look like. Improvisation has played a very significant role in almost all my research work.

Despite this, I have successfully guided through eight doctoral dissertations and several other interesting research projects. In total, I have published almost 100 articles in international publications and written chapters in seven international textbooks.

I worked at the university for 13 years as a clinic veterinarian. The post did not include research work. In fact, research was prohibited. It was only after a vote among the teachers' collegium that I was granted permission to do research work on the condition that it would not interfere with my other duties. During this period of time, I carried out most of my research at nights and weekends.

During the first few years I received support from Markus Sandholm, who was four years younger than me but was already a professor of pharmacology and had worked as a researcher in the United States. He was the only other person who was also interested in studying pets. He was a veritable treasure trove of ideas, and his knowledge of animal diseases and their treatment was exceptionally extensive. Markus was involved in a research project assessing how levels of pancreatic enzymes could be measured by means of agar gel diffusion, and whether the method could be used in the diagnosis of canine exocrine pancreatic insufficiency (EPI), a relatively common disease in dogs. Dogs with this disease do not secrete sufficient amounts of pancreatic enzymes into their intestines, resulting in maldigestion. These dogs eat large amounts of food but still lose weight.

At that time, there was no definitive method for diagnosing EPI. The agar gel diffusion method became my first research topic and later expanded to become the subject of my doctoral dissertation. I collected hundreds of stool samples from dogs to be analysed by Matti Järvinen in our laboratory. The results showed that there were no protease enzymes found in stool samples from dogs with EPI, whereas in healthy dogs, these enzymes were present, although not in every case. Thus, the problem was now how to differentiate healthy enzyme-secreting dogs from healthy dogs that did not secrete these enzymes. We called the latter ones "pseudo-EPI dogs". Markus came up with the idea of giving the dogs crude soya meal, which contains antiproteases. His thinking was that the antiproteases would inactivate the proteases present in the dogs' intestines, thus causing the pancreas to react by secreting large amounts of proteases into the intestines. In dogs with EPI, no proteases are found in stool samples even after they have been fed soya; however, they are found in both pseudo-EPI dogs and normal dogs. We developed the "crude soya test", in which crude soya was added to dog food, after which the dogs' stool samples were checked for proteases. The test was performed on more than one thousand dogs suspected of having EPI. Based on the results, we were able to state and based on this research it could be stated that the crude soya test is a reliable method for diagnosing EPI.

A few years after the publication of the crude soya test, the Englishmen developed the "TLI test", which allowed the proteases to be determined from blood samples. This test is as reliable as the crude soya test, but much easier to perform and therefore now widely used. I carried on with the EPI studies in two breeds in which EPI is a common disease, namely German Shepherds and Collies. Based on information extracted from the registers of the dogs with the disease I was able to state that EPI is possibly an autosomal, recessively inherited disease. However, it could also be a multi-gene disorder, in which case developing gene tests would be almost impossible.

In 1987, Alice Skonbäck contacted me and said that her German Shepherd, Dolly, had symptoms suggestive of EPI. Dolly's diagnosis was confirmed with the crude soya test. I told Alice that EPI is a 
hereditary disease and that dogs with it need treatment for the rest of their lives. Alice replied that she could not afford continuous medication. Surprisingly, she offered to give her dog to us in the hope that this would promote research into EPI. I thanked her and told her that we would have to make her donation the subject of a written agreement. Although I did not have any research ideas for Dolly at first, after thinking the matter over I came to the conclusion that we could use the dog in studies on medicines for the treatment of pancreatic disorders.

I had already been studying for some time with Professor Martti Marvola how differently sized tablets and capsules containing pancreatic enzymes are carried into the small intestine with food. For successful digestion, it is very important that the enzymes and the food are in the intestines at the same time. I decided to insert a fistula into Dolly's small intestine in order to obtain samples of bowel contents. There were no suitable fistulas for sale, but a precision engineer promised to make one out of stainless steel. The fistula was installed and it worked well. Throughout the study, we fed the dog the same food, adding different pancreatic enzyme medicines one at a time. After feeding the dog, we sampled her bowel contents via the fistula for eight hours. The lipase, amylase and protease concentrations in the samples were determined using the agar gel diffusion method. The results showed that the medication was most effective when the enzymes were added to the food in powdered form, or when raw pancreas was added to the dog's food. Commercially available tablets and capsules were considerably less effective.

After the study, I contacted Alice and told her that Dolly was in good health and had gained several kilograms. I suggested we would pay for Dolly's medicines for one year if she would take the dog back home. I told Alice that if we want to find out how EPI is inherited, we should cross two dogs with the disease and then see whether the puppies get ill. If the disease's inheritance follows the autosomal pattern, all the puppies would develop EPI. However, if the inheritance of EPI is controlled by several genes, only a few of the puppies would get sick. After a few days, Alice called me to say that she was willing to cross Dolly with a male with EPI, and she already had a promising candidate in mind. The offer sounded tempting but also frightening, because I had no funding or assistance for this kind of project. However, at the end of our discussion we decided to start the project and try to solve any problems as we went along.

I asked Maria Wiberg to participate in the Dolly project, and this marked the start of our collaboration. At that time, Maria was a fifthyear student, doing her advanced studies on the inheritance of EPI. Our collaboration lasted for a total of 15 years, during which time we published together 14 studies on EPI and Maria wrote her doctoral dissertation on EPI.

Dolly was crossed with a male dog with EPI and gave birth to five puppies. Alice was ready to take one of the puppies. She also knew two people who had previously had a dog with EPI, and two people who had had a German Shepherd. These people were potentially willing to participate in the study. After some discussion, all the people suggested by Alice promised to take a puppy. I explained to them both orally and in writing the nature of EPI and also told them that the dogs they were to take were highly likely to develop the disease. The owners were also given details about the purpose of the study and the kinds of tests that the dogs would undergo in the future. We also agreed that if the dogs were to develop EPI, their treatment would be free for the rest of their lives. The owners were not compensated for the upkeep costs of the dogs. Before giving the puppies to their new owners, Maria and I performed a laparotomy on each puppy, i.e. they were anaesthetised, their abdominal cavities were opened and a pancreas sample was obtained by ligation. During the next ten years, we performed this procedure 26 times. We did most of the operations on weekends, as the operating rooms were quieter then, and the dog owners had more time to bring their dogs in for surgery. The procedures were performed without any complications. Blood and stool samples were also taken from the dogs every few months. I stayed in close contact with the owners, and we gathered together for meetings quite often. During the entire study, which went on for 12 years, none of the owners ever asked for compensation for the dogs' transportation or other costs.

Based on our studies we were able to conclude that EPI is not a congenital disease. All the dogs had a normal pancreas when the laparotomies were first performed. One dog's pancreas began atrophying around the age of one, and after a few months, the dog showed the typical symptoms of EPI. After this, the dog spent the rest of its life on pancreatic enzyme medication, though its quality of life was fairly normal. Another dog's pancreatic atrophy began at the age of six. However, the atrophy halted when its pancreas was down to about one third of its original size. The dog had none of the clinical symptoms of the disease during its whole life, but tissue samples taken from its pancreas, as well as blood and stool samples, revealed partial pancreatic atrophy. The other dogs' pancreases remained completely normal. The samples taken during the study were cut into sections for histological and immunohistochemical tests. This meant that, for the first time, changes in the pancreas could be monitored as the atrophy progressed. These study results were an essential part of Maria's dissertation, in which she showed that EPI is an autoimmune disease. The point of the study was to determine the inheritance pattern of EPI in German Shepherds. Because only two of the dogs got ill, EPI could not be a single-gene disease. Instead, it had to be classified as a polygenic disease, requiring mutations in more than one gene and potentially also contributions from some environmental factors. The inheritance of EPI was also being intensively studied in the US during our Dolly project. Companies and researchers experienced in genetic research published three articles in which they stated that they were unable to find any specific gene that could cause EPI. Thus, they came to the same conclusion as that shown by our field test.

After giving birth, Dolly was healthy for six months, but suddenly her abdomen swelled up and her condition deteriorated. I got Dolly to the clinic, where she was immediately made surgery. We saw that the entire small intestine had twisted 180 degrees. After the surgery, Dolly only lived for a few hours. Volvulus of the small intestine was not unfamiliar to us. During the previous five years, we had had eight similar cases. All had been German Shepherds with EPI, and the condition proved fatal in all of them. Volvulus of the small intestine has not been reported anywhere else in the world, and textbooks mention that this condition is only found in Finland. 
Together with Maria, we also performed several studies to determine the most suitable and beneficial diet for dogs with EPI. This was of particular interest to dog food manufacturers, who had launched several special diets for dogs with EPI. However, our studies showed that none of the special foods were better than food meant for healthy dogs. We also found that there is great individual variation in dogs' reactions to foods, which means that the most suitable food must be determined by trial and error.

The first of my doctoral students to defend her dissertation was Irmeli Happonen. Her topic was to assess whether Helicobacter species were responsible for chronic vomiting symptoms in dogs. In human studies, research showing that $H$. pylori causes gastric and duodenal ulcers had attracted much attention. Several articles had also presented findings of Helicobacter species in dogs, but no detailed studies of their significance in dogs had been performed. Irmeli's studies showed that Helicobacter species occurred in practically all dogs, whether sick or healthy. There were several different species of the bacterium, but they were not the same as those found in humans. Marja-Liisa Hänninen identified the Helicobacter species and also isolated a new species about which nothing had previously been published.

The field tests performed by Irmeli showed that if the vomiting dogs were treated with medicines indicated for Helicobacter eradication, these bacteria were no longer seen immediately after the treatment. However, the lesions in the gastric mucosa remained unchanged, as did almost all the clinical symptoms. A few months after the medication had been discontinued, Helicobacter species were again found in the gastric mucosa. It was thus concluded that if Helicobacter species are found in a vomiting dog, it is not worthwhile to eradicate them.

In the 1980's, a molecular genetic method was developed for bacteria identification. Using this method, it was found that the intestinal microbiota contains an enormous number of different bacteria that could not be seen using culture methods. The microbiota is believed to influence a person's health and to cause intestinal disorders. This method also aroused interest in the treatment of pets, and Jan Suchodolski, working at Texas A\&M University, became the most famous researcher of this method. Researchers in Finland were also interested, but we had no plans about how to start canine studies. I came across an article by Maria Wilsson-Rahmberg describing how the pharmaceutical company Astra performs permanent small intestine fistula operations in dogs. The operation is rather complicated. A segment of the small intestine, about $15 \mathrm{~cm}$, is separated while maintaining its blood circulation. The segment is then invaginated, one end is attached to the small intestine and the other is taken through the abdominal wall and attached to the skin. This fistula does not require any metallic parts, and it can be used to drain bowel contents or to inject substances into the intestines via a tube. I phoned Maria in Sweden and invited her to Finland to teach how to perform fistula operations. Maria suspected that her employer would not allow this. However, after a while she notified me that she was in fact able to come and teach us some weekend. I asked Jaana Harmoinen and Minna Rinkinen to assist in the operations. Maria performed successful fistula operations on two test dogs. We monitored the dogs for a few weeks and found that the fistulas were functioning perfectly and the dogs did not react to them at all. Over the following years, Jaana and Minna successfully performed fistula operations on at least twenty dogs. The dogs lived with their fistulas for several years without any problems. I have not found any mention in the literature of the use of such fistulas in test dogs. We used the fistulated dogs in studies to find out how the antibiotic tylosin and changes in feeding influenced the microbiota of the small intestine. We fasted the dogs for five days and took samples of their bowel contents before, during and after the fast. We sent the bowel content samples to Texas for Jan to analyse.

In 1998, Lauri Jalkanen, representing a small, recently founded company, came to see me. He said they had a fantastic business idea but did not know how to implement it. At first, I did not understand what kind of idea this was and why he had come to see specifically me. Finally it became clear that the company, called Ipsat, was trying to develop a new form of treatment to decrease hospital-acquired infections caused by antibiotics. Some hospital-acquired infections develop because antibiotics change the intestinal microbiota so that it is no longer able to resist the growth of harmful bacteria. Antibiotic treatments are also responsible for the development of antibioticresistant bacterial strains in the intestinal flora. During intravenous antibiotic treatment, some of the antibiotics always end up in the bowel contents, causing changes in the intestinal flora. Hospital-acquired infections could be prevented by stopping antibiotics from entering the gut during parenteral antibiotic treatment. The idea developed by Ipsat was to administer oral beta-lactamase enzyme concomitantly with a parenteral antibiotic injection to break down antibiotics entering the intestines, thus preventing changes in the intestinal microbiota. Lauri Jalkanen asked me to suggest ways to study the use of their idea in dogs. I told him about our fistula dogs and suggested that it could be tested in them. After this meeting, Ipsat's CEO Kai Lindevall invited me for talks, which were to mark the beginning of collaboration lasting some ten years. I asked Jaana Harmoinen to join the project, and some years later it became the subject of her doctoral dissertation. The talks held with Ipsat were very frequent and full of challenges in spite of the collaboration working smoothly. We formed a plan to give the dogs intravenous ampicillin antibiotics shortly after oral beta-lactamase. We would sample bowel contents through the fistula for a few hours. The first thing to test was the form and the dose of beta-lactamase. We had also to assess when and how frequently to sample and when to feed the dogs. The results of the first series of tests showed that a certain beta-lactamase concentration could completely prevent ampicillin from entering the intestines. We were also able to show that beta-lactamase did not decrease circulatory ampicillin concentrations. No adverse effects of the enzyme were seen. For the second series of tests, we acquired $18 \mathrm{dogs}$ and divided them into three groups. The first group received both the antibiotic and the enzyme, the second group was given only the antibiotic, and the dogs in the third group received neither. The test went on for two weeks, and the dogs were fed and given the medicines four times daily. We found that the dogs on antibiotics only experienced changes in their intestinal flora together with increases in ampicillin-resistant $E$. coli strains as well as strains with TEM genes, which contribute to resistance. The dogs in the other groups did not experience any changes in their 
microbiota. The dogs in our study showed that ampicillin-induced changes in the intestinal flora can be prevented with beta-lactamase. Ipsat continued with the research and showed that beta-lactamase also acts similarly in humans. The company also developed a lactamase enzyme that prevents changes caused by many other antibiotics besides ampicillin. The lactamase project was expensive, and although Tekes and Sitra provided over ten million euros of funding, there was not enough money to start clinical studies in humans. Ipsat went bankrupt and sold the lactamase patent to a Swiss company for $€ 20,000$. This company sold it again to an American company, Synthetic Biologics, which at the moment (in 2015) has a market value of $€ 145$ million.

Tylosin belongs to the group of macrolide antibiotics. Millions of kilograms of tylosin have been used annually to promote the growth of pigs and cattle. The use of tylosin for this purpose was banned in Europe around 20 years ago. In Finland, it has been possible to purchase tylosin in tablet form for canine medication, although it has no official indications or dosing instructions. No studies on the efficacy of tylosin to treat canine diarrhoea had been published in the literature. Many Finnish dog owners were, however, convinced that tylosin is an efficient anti-diarrhoea agent for dogs. At the turn of the century, I began my first study on the efficacy of tylosin in the treatment of canine diarrhoea symptoms. I was assisted by two very hard-working students in the advanced stage of their studies, and the three of us performed the field study part, which lasted a few months. Fourteen dog owners, whose dogs had had diarrhoea problems for at least one year, participated in the study. I delivered freezers to the owners' homes in my car, and the students took many kinds of samples at specified intervals. The dog owners' patience was admirable, considering that during the study the dogs would quite often defecate in the owner's car or on the living room carpet. The study results showed that tylosin ends the diarrhoea symptoms within no more than three days, and the symptoms do not return as long as the medication is continued. However, in very many cases the symptoms return within one month of stopping the medication. Corticosteroid medication did not stop the diarrhoea symptoms within three days, and probiotics did not help prevent the recurrence of diarrhoea symptoms. We started calling the dogs in which tylosin did stop the diarrhoea symptoms as tylosinresponsive dogs (TRD). During the study, several different samples were taken from the dogs, but none of them explained why these dogs had diarrhoea symptoms.

The next tylosin study began unplanned. A dog called Paavo, who had continuous diarrhoea, had been with us for tests for over a year. Tests had failed to identify the cause of the diarrhoea, and the dog's general health was otherwise normal. Within a short period, six dogs in Paavo's section also got diarrhoea, and the symptoms persisted for over a month. At this point we started giving tylosin to all the dogs with diarrhoea. The symptoms lessened, but the stools did not become completely normal. We then stopped the medication and the diarrhoea symptoms worsened. The next step was to give the dogs three different antibiotics and corticosteroids each in turn, but these had no influence on the symptoms. After this, we changed the dogs' diets, which lessened but did not end the diarrhoea symptoms. Then we gave the dogs tylosin again for a week, and the diarrhoea symptoms stopped. The stools were monitored for three months, during which they remained normal in all dogs. What the study showed was that tylosin and diet have a synergistic effect. Pirkko Nokkala-Wahrman, an animal attendant, had a very large role in the performance of this study. She used a pictorial assessment table to estimate the consistency of every dog's every bowel movement throughout the study.

After this, I decided it was necessary to perform a double-blind study to show the efficacy of tylosin. No reports of double-blind studies in canine intestinal diseases had at that time been published in the literature. After lengthy discussions, the Finnish pharmaceuticals company Vetcare promised to start funding this study. It was a big decision for a smallish company. We agreed that Vetcare would pay a researcher's salary for three years, as well as a lot of other studyrelated costs. Susanne Kilpinen started as a doctoral student at this point, and she defended her doctoral dissertation on tylosin in the treatment of chronic canine diarrhoea seven years later. In order to achieve baseline characteristics as similar as possible for both the dogs on medication and the dogs on placebo in our double-blind study, we decided to only include dogs that had previously been treated successfully with tylosin for diarrhoea symptoms. To qualify for the registration phase, the dogs either had to be currently on tylosin or had had tylosin medication stopped within the last month. Almost fifty dog owners enrolled their dogs for the study. These dogs were taken off tylosin medication and monitored for any reappearance of diarrhoea symptoms. If the symptoms reappeared, the dogs were given either tylosin or placebo for one week. As a result, the symptoms disappeared in $79 \%$ of the dogs given tylosin vs. $28 \%$ of those given placebo. Dogs in which tylosin caused the diarrhoea symptoms to disappear then participated in a follow-up study to determine the recommended dose for the treatment of diarrhoea symptoms. It was found that dogs need considerably smaller amounts of tylosin than those recommended in the literature. During these two studies, several different samples were taken from the dogs to try to determine the basis of the efficacy of tylosin in the treatment of diarrhoea symptoms. Results from stool samples showed that tylosin significantly increased the levels of lactic acid bacteria and enterococci. It is possible that these bacteria have probiotic characteristics that have a positive effect in decreasing intestinal inflammation and diarrhoea symptoms.

My doctoral students Mairi Speeti, Minna Rinkinen and Rafael Frias conducted their dissertation work very independently. My role was simply that of an advisor. Their research was carried out in laboratories to a great extent and did not involve any clinical field studies.

The greatest source of joy in my studies has been the very competent doctoral students that I have been lucky enough to have. Even though study protocols tended to be very sketchy at the start of projects, the students always believed progress would be made. The many failures and changes to study protocols never discouraged them. Common to all my doctoral students has been their devotion to their research work and the fact that they contributed a lot of their own ideas and suggestions for improvements. We all worked together extremely well and over the years we became close friends. 
Another great source of help in my research work has been my advanced studies students. Several students contributed so significantly that they were cited among the authors of articles.
I would also like to thank the animal attendants and laboratory animal attendants who assisted me in sampling and carrying out other practical work.

Citation:

Elias Westermarck (2018) Challenges and Successes in Research Work. Integr J Vet Biosci Volume 2(3): 1-5. DOI: 10.31038/IJVB.1000112 